\title{
Paint adsorption on walls: I. Fractal-Stochastic model
}

\author{
Alberto A. Trejo-Franco, Eduardo Arvizu-Sánchez, Lisbeth A. Brandt-García, Yolanda G. \\ Aranda-Jiménez, Julio G. Lorenzo-Palomera, Edgardo J. Suárez-Dominguez* \\ FADU. Universidad Autónoma de Tamaulipas, México, Edgardo.suarez@docentes.uat.edu.mx
}

\begin{abstract}
Adsorption processes on surfaces is highly relevant in the industry since they allow specific characteristics to be obtained, from fixing materials such as paints to the efficiency of reactions on catalysts. Although this relationship can be estimated using the adsorption isotherms, there are practical problems associated with its obtention when it is about paint adsorption on walls: complex characteristics of the analyzed system since there is no fluid phase that is in contact with the surface (no concentration of adsorbate can be measured, as is done in traditional adsorption processes) In this case, a phase change occurs when the paint drying process takes place, so only the concentration of the pigment is known, and how it is distributed on the surface, this data is insufficient to build the adsorption isotherm. A stochastic model was obtained allowing the adsorption representation for coatings, estimating the equilibrium constant associated with the adsorption process and the distribution of active sites from the fractal dimension observed in the mesoscopic scale and adsorbate concentration at equilibrium. Two paint-coated surfaces were evaluated finding the adsorption value predicted from the use of the proposed model. From the morphology of the surface, predictive behaviors can be performed..
\end{abstract}

Key words : adsorption phenomena on walls, stochastic fractal model, adsorption-desorption isotherms.

\section{INTRODUCTION}

The application of paints on walls is essential for the protection of these surfaces and the conservation of residential structures. It is also applied to other kinds of products like plasters[1,2]. Covering power depends on paint composition and the pigment used [3], as well as on the porosity and roughness of the surface to be protected. This process is like the physical or chemical adsorption of an adsorbate (pigments and other paint components) on an adsorbent (wall surface).

From a physical chemistry point of view, the adsorption phenomena through the adsorption isotherms describe the equilibrium behavior of surface fraction, covered as a function of adsorbent concentration. In this case, for the microscopic scale, two fundamental processes take place: the adsorption of a molecule on a specific site or its desorption before the binding process with the active site. These processes occur randomly, which are manifested in the irregular patterns that adsorbate show on the surface and observed on the mesoscopic scale [4,5]. Its morphology is ultimately determined by the chemical nature of the adsorbate and the adsorbent and the dynamic processes that take place. These patterns are complicated to describe using Euclidean geometry, making the use of fractal geometry more plausible.

In the equilibrium state, the phenomenon is described through the adsorption isotherms [6], expressing relationship between concentration of adsorbate (in the gas or liquid phase) and the fraction of the surface covered by the adsorbate at a specified temperature, which is obtained from experimental behavior observed.

In the microscopic scale, the processes of adsorption and desorption occur randomly [7-8], which is manifested in the irregular patterns that form the adsorbate on the surface. These patterns are complicated to describe using Euclidean geometry, so it is more plausible to use fractal geometry [7]. Adsorption-desorption processes are highly relevant in the film fixing industry, obtaining materials, inorganic and organic synthesis, among others [8-10]. Although there are methods to obtain adsorption isotherms [10,11], it is necessary to deepen other versatile alternatives to get them and recognize the possible behaviors of this property.

Based on these initial considerations and the use of stochastic formalism based on the master equation, for the use in covering capacity of pigments used and paints, this paper proposes a mesoscopic model to describe the morphology behavior of these patterns as a function of the adsorption and desorption rate constants taking place on the microscopic scale. 


\section{METHOD}

\subsection{Model development}

In order to obtain a mesoscopic model to describe the behavior of the average and variance of the fraction of sites covered by adsorbate, the following assumptions are established:

i) formation of a single molecular layer of the adsorbate on the surface

occurs;

ii) the effect of neighboring molecules on the bond strength between adsorbate and adsorbent molecules is negligible;

iii) the extensive variable that describes the behavior of the system on the microscopic scale is the total amount of adsorbate $M$ molecules present in an area $\Omega$ of the surface of the solid;

iv) the intensive variable is the fraction of entire sites $\theta$ covered by the adsorbate, where the relationship between the intensive and extensive variable is given by:

$$
\theta=\frac{M a_{n}}{a}
$$

molecule;

where $a_{0}$ is the area occupied by an individual

v) the fluctuations associated with the concentration of adsorbate $C_{0} \mathrm{~mol} / \mathrm{m} 2$ expressed per unit area in the fluid phase that is in contact with the surface in the equilibrium state are assumed negligible, so that $C_{0}$ can be considered as variable deterministic;

vi) the probability of transition per unit of time associated with the adsorption process that takes place on the microscopic scale is established a priori, with the traditional equations of stochastic methods [12], such as:

$$
W_{M+1 / M}=\frac{k_{M}}{a} x_{0} N_{0}
$$

where $N_{0}$ is the total number of free sites of the area $a_{0}$ in which the adsorbate molecules can bind, $k_{a}$ is the adsorption rate constant and $x_{0}$ is the adsorbate molecules that are in the fluid phase;

vii) the probability of transition per unit of time that the desorption process occurs is established as:

$$
W_{M-1 / M}=k_{d} M
$$

where $k_{d}$ is the desorption rate constant. Taking into account that:

$$
N_{t}=N_{0}+M
$$

Equation 2 is written in the form:

$$
W_{M+1 / M}=\frac{k_{m}}{\Omega} x_{0}\left(N_{t}-M\right)
$$

such that the master equation describing the probability $P(M ; t)$ that at time $t$ the number of adsorbate molecules bound is equal to $M$ is:

$\frac{\partial P[M ; t)}{\partial t}=\left(E^{-1}-1\right) \frac{k_{m}}{\Omega} x_{0}\left(N_{t}-M\right) P(M ; t)+\left(E^{+1}-\right.$
$1) k_{d} M P(M ; t)$

subject to the initial condition:

$$
P\left(M_{0} ; 0\right)=1
$$

$E^{\alpha}$ being the ascent - descent operator that acts on the functions of discrete variables. If it is taken into account that when an individual microscopic process takes place, the change $\Delta M$ that occurs is negligible compared to $M$ then $M$ can be considered as a continuous variable, obtaining the Fokker-Planck equation:

$$
\begin{aligned}
& \frac{\partial P(M ; t)}{\partial t}=-\frac{\partial}{\partial M}\left[\frac{k_{a}}{\Omega} x_{0}\left(N_{t}-M\right)-k_{d} M\right] P(M ; t) \\
& +\frac{1}{2} \frac{\partial^{2}}{\partial M^{2}}\left[\frac{k_{m}}{\Omega} x_{0}\left(N_{t}-M\right)+k_{d} M\right] P(M ; t)
\end{aligned}
$$

Taking into account the relationship between the extensive and the extensive variable and that:

$$
P(M ; t) \partial M=P(\theta ; t) \partial \theta
$$

a change of variables is made in equation 8 so that it is expressed as a function of the intensive variable $\theta$ :

$$
\begin{gathered}
\frac{\partial P(\theta ; t)}{\partial t}=-\frac{\partial}{\partial \theta}\left[k_{\alpha} C_{0} N_{\alpha}(1-\theta)-k_{d} \theta\right] P(\theta ; t) \\
+\frac{1}{2}\left(\frac{\Omega_{0}}{\Omega}\right) \frac{\partial^{2}}{\partial \theta^{2}}\left[k_{\alpha} C_{0} N_{\alpha}(1-\theta)+k_{d} \theta\right] P(\theta ; t)
\end{gathered}
$$

where $N_{a}$ is Avogadro number; equation 10 is a linear Fokker-Planck equation, whose solution is a Gaussian probability function in which the expected value behaviors $\Phi$ and the variance $\sigma$ of $\theta$, respectively, are described through the system of temporal differential equations:

$$
\begin{gathered}
\frac{d \Phi}{d t}=k_{a} C_{0} N_{a}(1-\Phi)-k_{d} \Phi \\
\Phi(0)=\Phi_{0}
\end{gathered}
$$

$$
\begin{gathered}
\frac{d \sigma}{d t}=-2\left(k_{d}+C_{0} N_{\alpha} k_{a}\right) \sigma+\frac{a_{0}}{\Omega}\left(k_{\pi} C_{0} N_{\Pi}(1-\Phi)+k_{d} \Phi\right) \\
\sigma(0)=\sigma_{0} \ldots(12)
\end{gathered}
$$

In a steady-state, the temporal derivatives found on the left side of equations 11 and 12 are equal to 0 , obtaining: 
Alberto A. Trejo-Franco et al., International Journal of Emerging Trends in Engineering Research, 8(5), May 2020, 1667 - 1671

$$
\begin{aligned}
& \Phi=\frac{C_{0} K}{1+C_{n} K} \quad \ldots(13) \\
& \sigma=\frac{\underline{Q}_{0}}{\Omega} \frac{\left(\Phi-C_{0} K(\Phi-1)\right)}{2\left[1+C_{0} K^{2}\right)} \\
& P(\theta)=\frac{c}{\sqrt[2]{\sigma}} \exp \left(-\frac{[\theta-\varphi)^{2}}{2 \sigma}\right) .
\end{aligned}
$$

where $K_{K}=\frac{N_{\mu} k_{a x}}{k_{a d}}$ is proportional to the quotient between the adsorption and desorption rate constants and $C$ an integration constant that takes into account the range of possible values of $\theta$.

\subsection{Theoretical estimation of the fractal dimension}

According to the principles of fractal geometry, the content of the substance $S$ in a region of characteristic dimension $L$ is related to the fractal dimension of capacity $f$ according to the expression:

$$
S=g^{n-f_{L} f}
$$

where $L$ is the Euclidean distance between two points, $g$ represents the measurement precision, and $n$ is the dimension of the Euclidean space in which the fractal object is embedded. According to this expression, the fractal capacity dimension of an object immersed in a plane can be determined from a binary image of the object and the method of counting boxes [13]:

$$
f=\lim _{i \rightarrow 1} \frac{\ln q n}{\ln q}
$$

where $q$ is the number of non-dimensional cells $l$ in which the image is divided and $\eta_{0}$ the number of cells in which part of the object was observed, where $l=1$ is the smallest size of the cells.

To estimate the behavior of the fractal dimension as a function of $K$ and $C_{0}$, we considered an equilibrium state with the ergodicity property, which means that the expected value of the probability $\langle P(\theta)\rangle=\frac{c_{2}}{\sqrt[3]{\sigma}}$ is equivalent to the density $\rho$ of the adsorbate in an observed region of area $\Omega$. Considering equation $17, \rho$ is expressed as a potential function of $l=\Phi^{0.5}$.

$$
\rho=C_{\mathrm{g}} l^{-\mu}
$$

where:
If the amount of adsorbate $\lambda$ present in the mesoscopic scale is considered to be the product of the density $\rho$ and the area occupied $A=g^{2-f_{0} f_{0}}$ by the active sites, then:

$$
\lambda=C_{\mathrm{a}} l^{-\mu} \times g^{2-f_{1}} l^{f_{1}}
$$

From equation 20 and the definition of fractal dimension you get:

$$
f=1+\kappa C_{0} \quad \ldots(21)
$$

Equation 21 allows estimating the equilibrium constant associated with the adsorption process and the distribution of active sites from the fractal dimension observed in the mesoscopic scale and the concentration of adsorbate in the equilibrium.

\subsection{Fractal dimension analysis on surfaces.}

The proposed model to estimate the fractal dimension as a function of the adsorbate concentration was used to compare the coating capacity of two pigments A and B. To prepare the samples, the surface was covered with a liquid film in which the dye is found, and the samples were dried at a temperature of $28^{\circ} \mathrm{C}$ for 24 hours.

The surfaces were observed with a Konus College \# 5302 microscope, WF $15 \mathrm{x}$ eyepiece, with $10 \mathrm{X}$ magnification. The patterns were photographed with a SONY DSC-W530 Cyber Shot 14.1 megapixel camera, Carl Zeiss lens and 4X optical zoom with a resolution of 7 megapixels.

In total, a set of 21 images were treated, where the range of values considered for the observed adsorbate concentration was between 0 and 0.2 moles per $\mathrm{cm} 2$ for each of the pigments analyzed.

The patterns taken were determined by the fractal dimension per box count using the ImageJ v1.40g [14] program in which each color image is converted into an 8-bit image, from which a binary copy is obtained of the pattern to which the vertical and horizontal fractal dimension of the images is determined.

\section{RESULTS AND DISCUSSION}

The images, treated using the ImageJ program, setting a pixel intensity threshold equal to 166 for conversion to a binary image (Figure 1). The fractal dimension was determined by the box-counting method. In total, a set of 21 experiments were treated, where the range of values considered for the observed adsorbate concentration was between 0 and 0.2 moles per $\mathrm{cm} 2$. The experimental results obtained are shown in Figure 2.

$$
\begin{gathered}
\mu=\lim _{\Omega \rightarrow \Sigma_{0}} \lim _{l \rightarrow 1}\left(\frac{d \ln \sigma^{0.5}}{d l}\left(\frac{d \ln l}{d l}\right)^{-1}\right) \\
=1-\kappa C_{0} \ldots(19)
\end{gathered}
$$




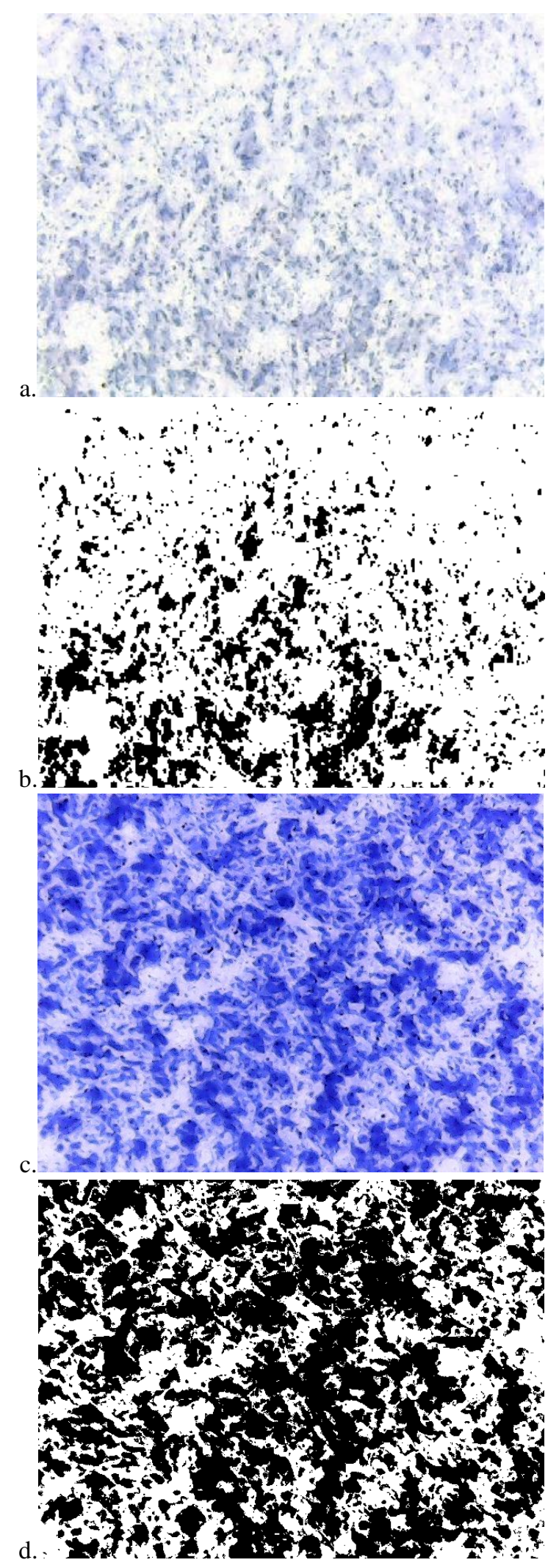

Figure 1. Original image (a. and c) and binary image ( $b$ and $d$ ) of the images of the surfaces covered by two types of pigments with two different concentrations a. and b. Pigment $\mathrm{A} \mathrm{Co}=0,17 f=1.5369 \mathrm{c}$. and d. Pigment $\mathrm{B}$ Co $=0,17 f=1.8780$

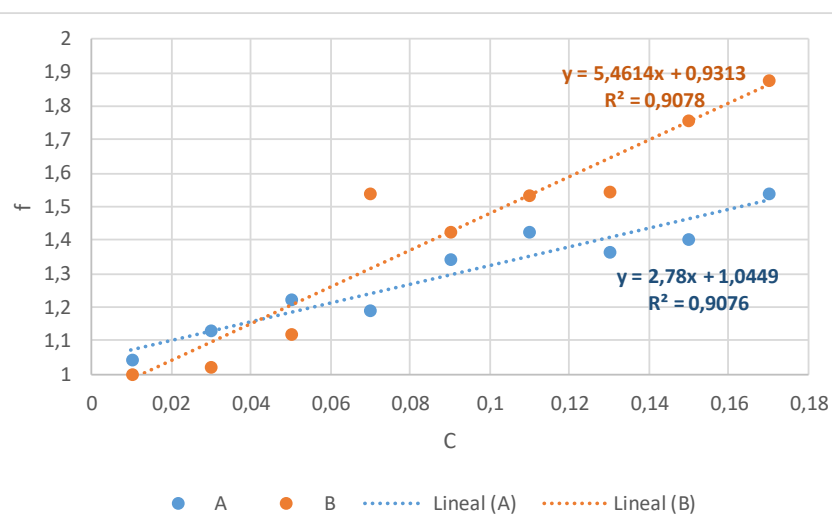

Figure 2. Behavior of the fractal dimension with respect to the concentration of pigments

In this case, for the same concentration of pigments, in case 1 $\mathrm{B}$ higher fractal dimension is obtained, from which it is inferred that pigment $\mathrm{B}$ has a better covering capacity than A.

With those results, it is possible to see that isotherms can be built with and stochastic fractal model and a relationship with image surface analysis. Model and analysis can be applied to other surfaces like presented in [15].

\section{CONCLUSION}

Covering capacity pigment depends on multiple factors as chemical-physical properties of the dye and the surface to be covered, as well as the molecular composition of the paint, temperature, and humidity of the environment so that conditions that affect the drying speed. The combined effect of these factors can be implicitly quantified through the relationship between the rate constants associated with the adsorption and desorption processes that take place on the microscopic scale during the paint application and drying process.

An equation was obtained that allows estimating the equilibrium constant associated with the adsorption process and the distribution of active sites from the fractal dimension observed in the mesoscopic scale and the concentration of adsorbate in the equilibrium. In this sense, the model proposed in this work allows the estimation of this constant from the observed fractal dimension and the concentration of the pigment in the paint, so that the adsorption process can be quantified without having to know the isotherm of adsorption.

\section{ACKNOWLEDGEMENT}

This research was partially supported by PROFEXCE program for FADU-UAT 2020.

EJSD thank to the Habana University and Dr. Izquierdo-Kulich for the theoretical and model revision . 


\section{REFERENCES}

1. Li, F., Wang, X., Guo, Q., Zhang, B., Pei, Q., \& Yang, S. (2019). Moisture Adsorption Mechanism of Earthen Plaster Containing Soluble Salts in the Mogao Grottoes of China. Studies in Conservation, 64(3), 159-173.

2. Zhang, H. Y., Yan, G. S., \& Wang, X. D. (2012). Laboratory test on moisture adsorption-desorption of wall paintings at Mogao Grottoes, China. Journal of Zhejiang University SCIENCE A, 13(3), 208-218. https://doi.org/10.1631/jzus.A1100204

3. Algrim, L. B., Pagonis, D., de Gouw, J. A., Jimenez, J. L., \& Ziemann, P. J. (2020). Measurements and Modeling of Absorptive Partitioning of Volatile Organic Compounds to Painted Surfaces. Indoor Air. https://doi.org/10.1111/ina.12654

4. Uddin, M. K. (2017). A review on the adsorption of heavy metals by clay minerals, with special focus on the past decade. Chemical Engineering Journal, 308, 438-462. https://doi.org/10.1016/j.cej.2016.09.029

5. Bhatnagar, A., \& Sillanpää, M. (2017). Removal of natural organic matter (NOM) and its constituents from water by adsorption-a review. Chemosphere, 166, 497-510.

6. Vanson, J. M., Boutin, A., Klotz, M., \& Coudert, F. X. (2017). Transport and adsorption under liquid flow: the role of pore geometry. Soft matter, 13(4), 875-885. https://doi.org/10.1039/C6SM02414A

7. Herrera-Hernández, E. C., Aguilar-Madera, C. G., Ocampo-Perez, R., Espinosa-Paredes, G., \& Núñez-López, M. (2019). Fractal continuum model for the adsorption-diffusion process. Chemical Engineering Science, 197, 98-108. https://doi.org/10.1016/j.ces.2018.11.058

8. Xu, F., Wang, T., Bohling, J., Maurice, A. M., Chen, H., Wu, L., \& Zhou, S. (2019). Insight into the dependence of dirt adsorption/desorption on the surface wetting behavior of $\mathrm{TiO} 2-$ based nanocomposite coatings. Progress in Organic Coatings, 131, 137-144. https://doi.org/10.1016/j.porgcoat.2019.02.015

9. Enea, D., Bellardita, M., Scalisi, P., Alaimo, G., \& Palmisano, L. (2019). Effects of weathering on the performance of self-cleaning photocatalytic paints. Cement and Concrete Composites, 96, 77-86. https://doi.org/10.1016/j.cemconcomp.2018.11.013

10. Albao, M. A. (2019). Impact of defect-driven surface inhomogeneity and minimal island formation on temperature-programmed desorption spectra: A kinetic Monte Carlo study. Surfaces and Interfaces, 16, 85-92.

11. Shumaker-Parry, J. S., \& Campbell, C. T. (2004). Quantitative methods for spatially resolved adsorption/desorption measurements in real time by surface plasmon resonance microscopy. Analytical chemistry, 76(4), 907-917.

https://doi.org/10.1021/ac034962a
12. Van Kampen, N. G. (1979). Composite stochastic processes. Physica A: Statistical Mechanics and its Applications, 96(3), 435-453. https://doi.org/10.1016/0378-4371(79)90005-0

13. Mandelbrot, B. B. (1983). The fractal geometry of nature (Vol. 173, p. 51). New York: WH freeman. https://doi.org/10.1119/1.13295

14. Schneider, C. A.; Rasband, W. S. \& Eliceiri, K.W. (2012), "NIH Image to ImageJ: 25 years of image analysis", Nature methods 9(7): 671-675, PMID 22930834. https://doi.org/10.1038/nmeth.2089

15. Talib, R., \& Sulieman, M. Z. (2016). Selected Melaka traditional houses: comprehensive analysis on building defects, failures and solutions including the Chetti house. IJETER, 4(8). 68-80 